\title{
CHANGES IN TEAR CYTOKINES FOLLOWING A SHORT PERIOD OF DAILY AND OVERNIGHT SILICONE HYDROGEL LENS WEAR
}

By Kim Duong, OD, MS, Cecilia Chao, PhD, BOptom, FAAO, Mark Willcox, PhD, FAAO FBCLA, FASM, FARVO, Kathryn Richdale OD, PhD, FAAO

\section{About the Authors}

At the time of this study, Kim Duong, Cecilia Chao, and Kathryn Richdale were with the College of Optometry, State University of New York, New York, USA. Cecilia Chao and Mark Willcox are with the School of Optometry and Vision Science, University of New South Wales, Sydney, Australia. Kim Doung is now with the University of Alabama and Kathryn Richdale is with the University of Houston.

Corresponding author: KRichdale@UH.edu

\section{ABSTRACT}

\section{Background and Objective}

To investigate changes in ocular surface inflammatory markers after daily and overnight silicone hydrogel contact lens wear in healthy wearers.

\section{Material and Methods}

Twenty-six subjects experienced with soft contact lenses were evaluated at baseline, after 1-day of silicone hydrogel lens wear, and after 1-night of wear. Basal tears were collected at each visit and tear cytokine concentrations were quantified using multiplex, bead-based [interleukin (IL)-1 $\beta$, IL-6, IL-10, IL12(p70), IL-17A and tumour necrosis factor (TNF)- $\alpha$ ] or ELISA (IL-8) kits. A historical control group of 27 non-contact lens wearers was used to compare absolute concentrations and diurnal variations in tear cytokine concentra-tions. Changes in cytokine concentrations were analyzed using linear mixed models. Linear regression with bootstrapping was used to assess whether changes in IL-1 $\beta$ concentrations were associated with changes in other cytokines.

\section{Results}

IL-8 concentrations decreased after 1 day of silicone hydrogel contact lens wear and returned to baseline levels the next morning $(p=0.04)$. This same diurnal fluctuation was seen in non-contact lens wearers $(p=0.03)$. With daily contact lens wear, there was a significant positive correlation between the changes in IL-1 $\beta$ and IL-8, TNF- $\alpha$, IL-10 and IL-12(p70) (all $p<0.03$ ). With overnight contact lens wear, there were significant positive correlations between the changes in IL-1 $\beta$ and IL-6, IL-17A and TNF- $\alpha$ (all $p<0.01$ ).

\section{Conclusion}

A short period of daily and overnight silicone hydrogel lens wear does not significantly alter the inflammatory status in adapted soft contact lens wearers.

Key Words: tear cytokines, silicone hydrogel contact lens wear, overnight wear, multiplex

Over 140 million people worldwide use soft contact lenses ${ }^{1}$ and it is expected that the number of contact lens wearers will continue to rise due to the increasing prevalence of refractive error and the fact that myopia control treatments may be delivered via soft contact lenses. ${ }^{2,3}$ Despite improvements in contact lens materials and solutions, contact lens wear is still associated with ocular surface inflammation and infection. In fact, overnight wear of silicone hydrogel lens materials have been identified as one of the primary risk factors for corneal infiltrative events. ${ }^{4-8}$

Cytokines are inflammatory mediators and are expressed by ocular surface cells and help maintain ocular health. ${ }^{9}$ However, changes to the normal production of inflammatory molecules during contact lens wear may predispose wearers to developing 
chronic or acute inflammation. The concentrations of cytokines in tears have been shown to change with routine daily wear of hydrogel contact and during contact lens-related inflammatory events. ${ }^{10-13}$ Some studies reported that the concentrations of interleukin (IL)-6 and IL-8 in tears increased during daily wear of hydrogel or silicone lenses. ${ }^{10,11}$ However, another study of silicone hydrogel lens wearers reported that the concentrations of IL- 6 and IL-8 did not change during daily wear of lenses, but there were reductions in the concentrations of IL-1 $\beta$ and IL-12(p70). ${ }^{14}$ To date, no study has examined changes in the concentration of cytokines in tears after overnight wear of silicone hydrogel lenses.

The primary aim of this study was to investigate change in cytokines in tears during a short period of daily and overnight wear of silicone hydrogel lenses in healthy adapted soft contact lens wearers. We also aimed to explore differences in individual responses to contact lens wear and relationships between various inflammatory mediators.

\section{METHODS}

\section{Prospective Study}

A prospective, single-site study was carried out between June 2014 and August 2015. The study was registered on ClinicalTrials.gov with the identifier NCT-02186431. The Institutional Review Board at the State University of New York, College of Optometry (SUNY Optometry) approved the protocol and the study followed the tenets of the Declaration of Helsinki of 1975 (revised in 2004). Written informed consent was obtained from all subjects before their participation in the study.

Subjects were recruited from SUNY Optometry and surrounding communities through flyers, email and web advertising. Subjects were full-time daily wear soft contact lens wearers (wore contact lenses for at least 1 year, 5 or more days per week, at least 6 hours per day, and denied overnight wear). Subjects were between the ages of 18 and 30 years. Exclusion criteria included astigmatism of more than 1.25 diopters, smoking, history of refractive surgery, self-diagnosed or practitioner diagnosed dry eye, history of ocular or systemic disease likely to affect the ocular surface (e.g., thyroid disease, diabetes), use of anti-inflammatory medications, punctual plugs, self-reported pregnancy during the study period, or significant corneal staining or ocular inflammation found at the baseline visit.

There were three study visits conducted within 24 hours: baseline with no lens wear (Visit 1), after 4 to 6 hours of daily lens wear (Visit 2), and after one full day and night of lens wear (Visit 3). Subjects were instructed not to wear contact lenses for at least 24 hours prior to Visit 1. Visits 1 and 3 were conducted between 8 am and $11 \mathrm{am}$, and Visit 2 was conducted between $12 \mathrm{pm}$ and $4 \mathrm{pm}$. During Visit 1, subjects' general health information and contact lens history were obtained. An anterior segment slit-lamp examination was conducted to confirm the health of the ocular surface, including lids, lashes, palperbral conjuctiva, tear film, caruncle and corneal and recorded as either normal or abnormal. The presence of corneal vascularization or corneal scar was also assessed. A slit lamp examination was also conducted at Visit 3 to document any changes due to continuous wear. After tear collection at Visits 1 and 3, sodium fluorescein dye (FUL-GLO, Moore Medical, Connecticut, USA) was instilled and staining was scored using the National Eye Institute/Industry (NEI) grading scale. ${ }^{15}$ The sum of all regions in both eyes was added to obtain a total score (range $0-30$ ).

At Visit 1, after tear collection and ocular surface examination, subjects were fitted with Senofilcon A contact lenses (Acuvue Oasys, base curve $8.4 \mathrm{~mm}$, Johnson \& Johnson Vision Care, Jacksonville, FL) bilaterally and were asked to continuously wear the contact lenses until Visit 3. Senofilcon A material was selected because it is one of the most commonly prescribed silicone hydrogel lens materials in the United States and is FDA approved for overnight wear. ${ }^{16}$

At all three visits, non-stimulated tears were collected from both eyes using glass capillary tubes (Blaubrand intraMARK; BRAND GMBH, Wertheim, Germany) ${ }^{17}$ A total of $10-20 \mu \mathrm{L}$ tears were collected. To ensure that basal tears were collected, only tears with a flow rate of less than approximately $3 \mu \mathrm{L}$ per minute were kept. ${ }^{18}$ Tears were centrifuged to remove debris and stored in a $-80^{\circ} \mathrm{C}$ freezer until analysis. Custom multiplex assays (X-plex format; Human cytokine group 1: IL-1 $\beta$, IL-6, IL- 10,

J Cont Lens Res Sci Vol 1(1):3-21; September 29, 2017.

(c) 2017 Journal of Contact Lens Science and Research. All rights reserved. This article is distributed under the terms of the Creative Commons Attribution-Non Commercial 4.0 International License. 
IL-12p70, IL-17A, TNF- $\alpha$; Bio-Rad Laboratories Inc., Hercules, CA) were used to analyze the tear samples following the manufacturers' protocols and as previously described ${ }^{19}$ using a 1:10 dilution in PBS. ${ }^{8,9}$ Due to the higher concentrations of IL-8 in tears, a Human IL-8 UltraSensitive ELISA Kit (Invitrogen Corporation, CA; detection limits: $<100 \mathrm{fg} / \mathrm{mL}$ ) was used to quantify IL-8 in tears after a 1:200 dilution in sample diluent. Samples were tested in duplicate for both the multiplex and standard ELISA assays if tear volumes were sufficient. To determine recovery rates of cytokines in samples, a previously published method was used. ${ }^{17}$ The recovery rate of each cytokine was calculated to determine the reliability to detect cytokines using the following equation ( $\mathrm{A}=$ concentration of known standard and in tears; $\mathrm{B}=$ concentration of known standard; $\mathrm{C}=$ concentration in tear sample): $:^{14,17}$

$$
\text { Recovery Rate }=A /(B \times 108+C \times 12) / 120) \times 100 \%
$$

Based on data from a previous study ${ }^{14}$ a sample size of 25 was required to provide sufficient power (Power $=0.8, \alpha=0.05$ ) to detect a $20 \%$ difference in the concentration in tears of IL-6, IL-8, IL-12(p70) or TNF- $\alpha$ between study visits.

\section{Historical Control Group}

In order to examine the effect of contact lens wear distinct from typical diurnal fluctuations, we utilized data from our previous study. ${ }^{14}$ The historical control group was comprised of 27 non-contact lens wearers with mean age of $25.5 \pm 8.5$ years; $60 \%$ of subjects were female. As in the prospective study, non-stimulated tears were collected in the morning and evening using glass capillary tubes. ${ }^{14}$ Tear cytokine concentrations in the historical study were also analyzed using multiplex assays (Bio-Plex, 27-plex panel, including IL-1 $\beta$, IL-6, IL-8, IL- 10, IL-12p70, IL-17A, TNF- $\alpha$, Bio-Rad Laboratories, Inc, Hercules, CA).

\section{Statistical Analysis}

Data were analyzed using SPSS version 22 (SPSS for Mac, Chicago, IL). Descriptive statistics were reported as mean \pm standard deviation or median and range, as appropriate. Tear cytokine concentrations were calculated and compared for Visit 1 and 2 (effect of daily wear) and Visit 1 and 3 (effect of overnight wear) using linear mixed models. Post-hoc comparisons were carried out with Visit 1 as the reference category using a least significant difference (LSD) adjustment for multiple comparisons. Mann-Whitney U test was used to assess differences in tear cytokine concentrations between the current contact lens wear cohort and our historical non-lens wear controls. Wilcoxon Signed Ranks tests were carried out to examine the changes in corneal staining between Visit 1 and 3 and to compare diurnal changes in tear cytokine concentration in historical control group.

To explore the role of IL-1 $\beta$, as a key orchestrator of the inflammatory response, ${ }^{20,21}$ IL- $1 \beta$ was assigned as the independent variable for linear regression to assess the likelihood that the concentration of other cytokines (dependent cytokines) would also change as the concentration of IL- $1 \beta$ changed. To account for skewed distribution in some variables, $90 \%$ confidence intervals were determined by bootstrapping. The results of the linear regression are reported as the regression coefficients ( \pm standard error), $p$-values and adjusted $\mathrm{R}^{2}$ values. Participants with values in the $80^{\text {th }}$ percentile of the distribution for both independent and dependent cytokines were also reported. Results were considered significant when $p$-values were less than 0.05 .

\section{RESULTS}

A total of 26 subjects completed the prospective study, of which 5 were men and 21 women (aged 23.7 \pm 1.5 years). Subjects reported an average contact lens washout time of approximately two days $(43.5 \pm 14.0$ hours) prior to Visit 1 . The average daily wear time was $5.1 \pm 0.7$ hours prior to Visit 2. Overnight wear time was $7.1 \pm 1.4$ hours. Subjects reported to Visit 3 approximately $1.9 \pm 1.3$ hours after awakening and had an average total wear time of $23.1 \pm 0.4$ hours (21.6 - 24.0 hours) from Visit 1 to Visit 3. All subjects that were included in the study had no significant anterior slit lamp findings at both visits. Corneal staining was not significantly different between Visit $1[0(0-2)]$ and Visit 3 [1.0-(0-3)] $(\mathrm{p}=0.15)$. No adverse events were reported in the study.

J Cont Lens Res Sci Vol 1(1):3-21; September 29, 2017.

(C) 2017 Journal of Contact Lens Science and Research. All rights reserved. This article is distributed under the terms of the Creative Commons Attribution-Non Commercial 4.0 International License. 
All subjects were established ( $\geq 1$ year of wear) and regular ( $\geq 4$ days/week) soft CL wearers. Of the 26 subjects, 9 subjects wore monthly lenses (AirOp-tix and Biofi nity), 9 wore biweekly lenses (Acuvue Oasys and Acuvue Advance), and 8 wore daily disposable lenses (Acuvue Moist, Acuvue True Eye, Proclear 1-Day, Dailies Total 1). Of the subjects that wore reusable lenses, 12 used multipurpose solution (Opti-Free, Biotrue and generic brands), 5 used hydrogen peroxide solution and one subject used a combination of hydrogen peroxide and multi-purpose solution.

All six cytokines could be detected in the samples of the majority of subjects (range: $91-100 \%$ of samples). The cytokine recovery rates ranged between 71 and $115 \%$ for all cytokines, similar to previously reported data using multiplex assays. ${ }^{17}$ The absolute concentration of the tear cytokines are presented in Table 1. The concentration of IL-8 changed significantly over time (overall $p=0.04$ ), with a decrease of approximately $50 \%$ after one day of wear (Visit 1 to Visit 2, $p=0.04$ ) and returned to baseline after one night of wear (Visit 1 to Visit $3, p=0.79$ ). The concentration of the other cytokines in tears was not significantly different from baseline (Visit 1) after either one day (Visit 2) or one night (Visit 3) of continuous lens wear (all $p \geq 0.06$ ).
Comparing the prospective contact lens wear group to the historical non-lens wear controls, there were no significant differences in sex $(p=0.66)$ or age $(p=0.80)$. There were no significant differences between non-lens wearers' and lens wearers' morning tear cytokine concentrations (Table 1, CL Visit 1 vs. NCL AM), except for IL-10, which was higher in the contact lens wear group $(p<0.001)$. Similarly, only IL-8 $(p=0.02)$ and IL-10 $(p=0.02)$ concentrations were different in the evening tear samples (Table 1, CL Visit 2 vs. NCL PM). IL-10 was again higher in contact lens wearers but IL-8 was higher in the non-lens wear group. IL-8 decreased by approximately the same percentage $(50 \%)$ from morning to evening in both non-contact lens and contact lens wearers (Table 1).

There were significant correlations between the change in the concentration of IL- $1 \beta$ and the change in concentrations of IL-8, IL-10, IL-12(p70) and TNF- $\alpha$ after daily wear (Table 2 ). For overnight wear, there were significant correlations between the change in the concentration of IL-1 $\beta$ and IL-6, IL-17A and TNF- $\alpha$ (Table 2). The significant correlations (adjusted $\mathrm{R}^{2}$ ) ranged from 0.22 to 0.66 , with IL-12p70 and 17A showing the highest correlations. With daily wear, two participants with changes in the $80^{\text {th }}$ or

Table 1. Absolute Concentrations of Cytokines (Pg/Ml) for the Prospective Study of Contact Lens Wearers and Historical Control Group of Non-contact Lens Wearers. $P$-Values Indicate Differences between Study Visits.

\begin{tabular}{|c|c|c|c|c|c|c|c|}
\hline Cytokine & $\begin{array}{c}\text { CL Visit 1 } \\
(\mathbf{A M})\end{array}$ & $\begin{array}{c}\text { CL Visit 2 } \\
(\mathbf{P M})\end{array}$ & $\begin{array}{c}\text { CL Visit } \mathbf{3} \\
(\mathbf{A M})\end{array}$ & $\boldsymbol{p}$-values+ & $\begin{array}{c}\text { NCL } \\
(\mathbf{A M})\end{array}$ & $\begin{array}{c}\text { NCL } \\
(\mathbf{P M})\end{array}$ & $\boldsymbol{p}_{\text {-values^ }}$ \\
\hline IL-8 & $\mathbf{4 1 8} \pm \mathbf{5 3 8}$ & $\mathbf{2 0 8} \pm \mathbf{1 9 8}$ & $\mathbf{4 4 5} \pm \mathbf{3 4 7}$ & $\mathbf{0 . 0 4}$ & $\mathbf{8 8 6} \pm \mathbf{7 7 5}$ & $\mathbf{4 0 0} \pm \mathbf{2 3 3}$ & $\mathbf{0 . 0 3}$ \\
\hline IL-1 $\beta$ & $29 \pm 15$ & $32 \pm 17$ & $27 \pm 15$ & 0.47 & $30 \pm 13$ & $32 \pm 18$ & 0.78 \\
\hline IL-6 & $77 \pm 62$ & $75 \pm 57$ & $81 \pm 86$ & 0.58 & $75 \pm 43$ & $92 \pm 48$ & 0.54 \\
\hline IL-10 & $54 \pm 43$ & $50 \pm 37$ & $56 \pm 41$ & 0.54 & $17 \pm 11$ & $24 \pm 16$ & 0.30 \\
\hline IL-12(p70) & $151 \pm 81$ & $161 \pm 86$ & $149 \pm 74$ & 0.76 & $180 \pm 100$ & $219 \pm 152$ & 0.64 \\
\hline IL-17A & $234 \pm 173$ & $294 \pm 246$ & $202 \pm 137$ & 0.06 & $172 \pm 136$ & $222 \pm 183$ & 0.64 \\
\hline TNF- $\alpha$ & $228 \pm 144$ & $293 \pm 212$ & $247 \pm 206$ & 0.35 & $239 \pm 192$ & $299 \pm 248$ & 0.68 \\
\hline
\end{tabular}

$I L=$ Interleukin, $T N F=\alpha$ : tumour necrosis factor- $\alpha ; C L=$ contact lens wearers in this current cohort NCL $=$ non-contact lens wearers in our previous study; $A M=$ morning measurement; $P M=$ evening measurement; + = comparison between $C L V 1,2$ and 3;

$\wedge=$ comparison between NCL AM and PM. Bold indicates significant levels of 0.05 .

J Cont Lens Res Sci Vol 1(1):3-21; September 29, 2017.

(C) 2017 Journal of Contact Lens Science and Research. All rights reserved. This article is distributed under the terms of the Creative Commons Attribution-Non Commercial 4.0 International License. 
greater percentile for IL- $1 \beta$ also had changes to IL-8, IL-12p70 and TNF- $\alpha$ (subjects 4 and 8; Table 2). With overnight wear, two participants had increases above the $80^{\text {th }}$ percentile in the concentration of IL$1 \beta$ and IL- 6 and IL-17A (subject 8 and 25).

\section{DISCUSSION}

This study found little change in tear cytokines after nearly 24 hours of continuous silicone hydrogel contact lens wear. The levels of tear cytokines in contact lens wearers were generally consistent with our historical control group of non-contact lens wearers, indicating that a short term of daily and overnight wear may not significantly alter the inflammatory status of the ocular surface in healthy established wearers. This study also provided evidence of the relationship between IL-1 $\beta$, a key orchestrator of the inflammatory response, ${ }^{20-22}$ and other tear cytokines and highlighted some potential differences in individual responses.

The concentration of IL-8 in tears decreased after one day of contact lens wear (Visit 2) and returned to baseline levels the next morning (Visit 3). The same diurnal fluctuation was noted in our non-contact lens control group. Uchino et al. ${ }^{23}$ reported that the concentration of IL-8 in tears of healthy non-contact lens wearers remained constant throughout the day, but Thakur et al. ${ }^{24}$ found that IL-8 significantly increased during sleep in adapted contact lens wearers, which agrees with our finding of higher levels at Visit 3. IL-8 is a chemokine for neutrophils, thus a diurnal fluctuation in IL-8 is part of the body's natural defense of the ocular surface during sleep. ${ }^{9,25}$ This study found that the concentrations of other tear cytokines did not change significantly after daily or overnight silicone hydrogel contact lens wear. Data from the historical non-contact lens wearers group suggest that there are no other expected diurnal fluctuations in the cytokines we studied.

Thakur et al. ${ }^{24}$ reported no significant change in the concentration of IL- 6 in tears after sleeping in hydrogel contact lenses, similar to the findings in this study. However, Poyraz et $\mathrm{al}^{11}$ reported increased concentrations of IL-6 in tears with daily wear of silicone hydrogel lenses. It's important to note that

Table 2. Relationships Between IL-1 $\beta$ Concentration and Other Cytokines

\begin{tabular}{|c|c|c|c|c|c|}
\hline $\begin{array}{l}\text { Independent } \\
\text { Cytokine }\end{array}$ & $\begin{array}{c}\text { Dependent } \\
\text { Cytokines }\end{array}$ & $p$-value & $\begin{array}{c}\text { Regression } \\
\text { coefficient } \\
\text { (standard error) }\end{array}$ & $\begin{array}{c}\text { Adjusted } \\
\qquad \mathbf{R}^{2}\end{array}$ & $\begin{array}{l}\text { Subject (study number) } \\
\text { with concentrations } \\
\text { above } 80^{\text {th }} \text { percentile for } \\
\text { both cytokines }\end{array}$ \\
\hline \multirow{6}{*}{$\begin{array}{l}\text { IL-1 } \beta \\
\text { concentration in } \\
\text { tears collected after } \\
\text { daily wear of lenses }\end{array}$} & IL-8 & 0.03 & $0.33(0.14)$ & 0.22 & 4,8 \\
\hline & IL-6 & 0.29 & $0.11(0.10)$ & 0.14 & - \\
\hline & IL-10 & $<0.001$ & $1.50(0.20)$ & 0.58 & 4,20 \\
\hline & IL-12(p70) & $<0.001$ & $2.47(0.31)$ & 0.62 & 4,8 \\
\hline & IL-17A & 0.20 & $0.54(0.40)$ & 0.14 & - \\
\hline & TNF- $\alpha$ & $<0.001$ & $0.57(0.15)$ & 0.39 & 4,8 \\
\hline \multirow{6}{*}{$\begin{array}{l}\text { IL-1 } \beta \\
\text { concentration in } \\
\text { tears collected after } \\
\text { overnight wear of } \\
\text { lenses }\end{array}$} & IL-8 & 0.64 & $-0.15(0.31)$ & 0.04 & - \\
\hline & IL-6 & 0.01 & $0.87(0.31)$ & 0.30 & 8,25 \\
\hline & IL-10 & 0.13 & $0.86(0.55)$ & 0.17 & - \\
\hline & IL-12(p70) & 0.32 & $1.37(1.35)$ & 0.19 & - \\
\hline & IL-17A & $<0.001$ & $2.17(0.34)$ & 0.64 & $8,19,25$ \\
\hline & TNF- $\alpha$ & $<0.001$ & $1.36(0.34)$ & 0.46 & 25 \\
\hline
\end{tabular}

$I L=$ Interleukin; TNF- $\alpha=$ tumour necrosis factor $-\alpha$.

J Cont Lens Res Sci Vol 1(1):3-21; September 29, 2017.

(C) 2017 Journal of Contact Lens Science and Research. All rights reserved. This article is distributed under the terms of the Creative Commons Attribution-Non Commercial 4.0 International License. 
Poyraz et $\mathrm{al}^{11}$ examined neophyte lens wearers over the first six months of lens wear, whereas the current study used subjects who had at least one year of lens wear experience.

Other factors have also been reported to be different in the first 6 to 12 months of lens wear, including the binding of Pseudomonas aeruginosa to exfoliated corneal epithelial cells ${ }^{26}$ (greater for up to six months of lens wear then returning to baseline levels) and risk of experiencing microbial keratitis (higher risk in the first six months of lens wear). ${ }^{4}$

Differences in tear cytokine concentrations between lens wearers and non-lens wearers could not be found in this study. Our findings are consistent with Dogru et $\mathrm{al}^{27}$ which found no significant differences between IL-8 or IL-10 in neophytes at baseline versus two weeks of daily hydrogel contact lens wear. Willcox et al. ${ }^{14}$ reported a decrease in the concentration of IL-1 $\beta$ and IL-12(p70) after daily disposable silicone hydrogel lenses wear. Willcox et al. ${ }^{14}$ also reported a decrease in concentrations of TNF- $\alpha$ with daily wear of silicone hydrogel lenses compared to concentrations during the no-contact lens stage. Again, it is important to note that Dogru et $\mathrm{al}^{27}$ examined only neophyte lens wearers, Willcox et al. ${ }^{14}$ examined a combination of neophytes and established lens wearers, while the current study examined only established lens wearers. To our knowledge, this is the first study to examine diurnal variation and the differences in tear concentrations of IL- 17A in soft contact lens wearers and non-wearers. Taken together, these studies suggest that further research is needed to examine subjects as they first enter into daily and extended lens wear and then become adapted lens wearers so that we may better understand the initial ocular response and adaptation to contact lens wear.

In addition to understanding the general ocular inflammatory response, it is also important to understand individual differences in the inflammatory response. The results of this study suggest that certain individuals may respond with a much larger change of tear cytokines as a result of contact lens wear. Genetic studies by Carnt et al. ${ }^{28,29}$ and Keijser et al. ${ }^{30}$ have shown that differences in certain single nucleotide polymorphisms (SNPs) of IL-6, IL-10 and IL-12(p70) were associated with increased susceptibility and severity of keratitis. This is the first study to report that some individuals may also have increased levels of cytokines produced at the ocular surface and this increase may be driven by the production of IL-1 $\beta$. Two participants had much higher concentrations of IL-1 $\beta$ and IL-8, IL-12(p70) and TNF- $\alpha$ with daily wear, and two participants had higher concentrations of IL-1 $\beta$, IL-6, IL-17A and TNF- $\alpha$ with overnight wear. A higher correlation of IL- $1 \beta$ and TNF- $\alpha$ in daily and overnight wear may suggest an overall greater inflammatory response since IL-8, IL-12(p70) and TNF- $\alpha$ are also pro-inflammatory mediators. ${ }^{29,31} \mathrm{~A}$ higher correlation of IL-1 $\beta$ and IL-17A in overnight wear but not daily wear is likely because of the role of IL-17 on initiation and modulation of neutrophils, which increased significantly during sleep, on the ocular surface apart from modulating proinflammatory responses. ${ }^{32,33}$ These high responders were not outliers in their baseline measurements (Visit 1), it is only their change in cytokine levels following contact lens wear that were exceptionally high. It could be informative to perform studies on subjects with a history of corneal infiltrative events to explore the hypothesis that patients with higher inflammatory responses may be more likely to experience contact lens-related inflammatory complications.

The washout period needed to return a person's tear level to "baseline" from contact lens wear is unknown. A washout period of 1 day to 1 month has been used in contact lens-related studies..$^{14,34,35}$ Our group has previously shown that 1 week washout of contact lens wear did not affect the examined tear cytokine concentrations. ${ }^{36}$ Although more than a month washout period may be required for the inflammatory cytokines return to pre-contact lens wear level. It was not feasible to have subjects refrain from CL wear for months. Research in animal models described the complex process of corneal epithelial wound healing in three stages: the first two are complete in under 36 hours and the third stage can take months. The average washout time in this study corresponded to expected completion of stage 1 and 2 , and there was little to no epithelial damage seen at Visit 1 for all subjects.

J Cont Lens Res Sci Vol 1(1):3-21; September 29, 2017.

(C) 2017 Journal of Contact Lens Science and Research. All rights reserved. This article is distributed under the terms of the Creative Commons Attribution-Non Commercial 4.0 International License. 
A limitation of this study is the use of a historical non-contact lens wearer control group. Tear cytokine concentrations can vary across studies due to the use of different techniques, including different analysis platforms and collection methods. ${ }^{10,11,36}$ Although collection methods were the same, the use of a multiplex versus ELISA assays could have magnified differences in IL-8 concentrations seen between contact lens wearers in the prospective cohort and non-contact lens wearers in the historical cohort. Future studies are warranted to examine potential differences in IL-8 and IL-10 between non-contact lens wearers and contact lens wearers. Furthermore, this study only subjectively indicated the presence of abnormal or normal slit lamp findings at Visit 1 and Visit 3. Future studies should include quantitative data such as Schirmer's value and giant papillary conjunctivitis grading to describe the ocular surface status.

\section{CONCLUSION}

This study suggests that, for established healthy contact lens wearers, short-term silicone hydrogel daily or overnight wear does not have a significant effect on ocular surface inflammatory status. Future studies are needed to explore potential changes in other inflammatory biomarkers, and individual responses to contact lens wear including those wearers with a history of inflammatory events. A better understanding of both the general and individual immunological response of the ocular surface to soft contact lens wear could help identify wearers at higher risk for complications.

\section{ACKNOWLEDGEMENTS}

Parts of the results of the study have been reported as an abstract at the American Academy of Optometry meeting in New Orleans, LA, October 7, 2015.

We would like to acknowledge the equipment and technical support from Mr. Bob Laffer from Bio-Rad Laboratories. We would also like to acknowledge Kim Duong's Master's Thesis Committee, Dr. Stewart Bloomfield and Dr. Miduturu Srinivas, at the State University of New York, for their support of her project. Finally, we gratefully acknowledge Dr. Nancy Briggs, at the University of New South Wales, for her help with statistical analysis.

\section{DISCLOSURE OF POTENTIAL CONFLICT OF INTEREST}

All authors have no affiliations with or involvement in any organization or entity with any financial interest or non-financial interest in the subject matter or materials discussed in this manuscript.

\section{DISCLOSURE OF FUNDING SOURCE}

This study was partially supported by an NIH/NEI T35 grant (EY020481, KD).

\section{REFERENCES}

1. Cope JR, Collier SA, Rao MM, et al. Contact lens wearer demographics and risk behaviors for contact lens-related eye infections--United States, 2014. MMWR Morb Mortal Weekly Rept 2015;64(32):865-70.

2. Anstice NS, Phillips JR. Effect of dual-focus soft contact lens wear on axial myopia progression in children. Ophthalmology 2011;118(6):1152-61.

3. Sankaridurg P, Holden B, Smith E, 3rd, et al. Decrease in rate of myopia progression with a contact lens designed to reduce relative peripheral hyperopia: one-year results. Investig Ophthalmol Vis Sci 2011;52(13):9362-67.

4. Stapleton F, Keay L, Edwards K, et al. The incidence of contact lens-related microbial keratitis in Australia. Ophthalmology 2008;115(10):1655-62.

5. Chalmers RL, Keay L, McNally J, Kern J. Multicenter case-control study of the role of lens materials and care products on the development of corneal infiltrates. Opto Vision Sci 2012;89(3):316-25.

6. Radford CF, Minassian D, Dart JK, Stapleton F, Verma S. Risk factors for nonulcerative contact lens complications in an ophthalmic accident and emergency department: a case-control study. Ophthalmology 2009;116(3):385-92.

7. Chalmers RL, Keay L, Long B, Bergenske P, Giles T, Bullimore MA. Risk factors for contact lens complications in US clinical practices. Opto Vision Sci 2010;87(10):725-35.

8. Chalmers RL, Wagner H, Mitchell GL, et al. Age and other risk factors for corneal infiltrative and inflammatory events in young soft contact lens wearers from the Contact Lens Assessment in Youth (CLAY) study. Investig Ophthalmol Vis Science 2011;52(9):6690-96.

J Cont Lens Res Sci Vol 1(1):3-21; September 29, 2017.

(C) 2017 Journal of Contact Lens Science and Research. All rights reserved. This article is distributed under the terms of the Creative Commons Attribution-Non Commercial 4.0 International License. 
9. Thakur A, Willcox MD, Stapleton F. The proinflammatory cytokines and arachidonic acid metabolites in human overnight tears: homeostatic mechanisms. J Clin Immunol 1998;18(1):61-70.

10. Schultz CL, Kunert KS. Interleukin-6 levels in tears of contact lens wearers. J Interfer Cytok Res 2000;20(3):309-310.

11. Poyraz C, Irkec M, Mocan MC. Elevated tear interleukin-6 and interleukin-8 levels associated with silicone hydrogel and conventional hydrogel contact lens wear. Eye Cont Lens 2012;38(3):146-49.

12. Lema I, Duran JA, Ruiz C, Diez-Feijoo E, Acera A, Merayo J. Inflammatory response to contact lenses in patients with keratoconus compared with myopic subjects. Cornea 2008;27(7):758-63.

13. Thakur A, Willcox MD. Cytokine and lipid inflammatory mediator profile of human tears during contact lens associated inflammatory diseases. Exper Eye Res 1998;67(1):9-19.

14. Willcox MD, Zhao Z, Naduvilath T, Lazon de la Jara P. Cytokine changes in tears and relationship to contact lens discomfort. Molecul Vis 2015;21:293-305.

15. Lemp MA. Report of the National Eye Institute/Industry workshop on Clinical Trials in Dry Eyes. CLAO 1995;21(4):221-32.

16. Johnson\&Johnson Vision care. ACUVUE® OASYSTM Brand Contact Lenses with HYDRACLEAR ${ }^{\circledR}$ Plus. Package Insert. http://www.acuvue.com/sites/default/ files/jjvc-media_enCA/pdf/package-insert-acuvueoasysastigmatism.pdf.

17. Liu J, Shi B, He S, Yao X, Willcox MD, Zhao Z. Changes to tear cytokines of type 2 diabetic patients with or without retinopathy. Molecul Vis 2010;16:2931-38.

18. Fullard RJ, Snyder C. Protein levels in nonstimulated and stimulated tears of normal human subjects. Investig Ophthalmol Vis Sci 1990;31(6):1119-26.

19. Chao C, Golebiowski B, Stapleton F, Richdale K. Changes in tear cytokine concentrations follwoing discontinuaton of soft contact lenses - a pilot study. Eye Contact Lens 2016 Jul;42(4):237-43. doi: 10.1097/ ICL.0000000000000182.

20. Hazlett LD. Role of innate and adaptive immunity in the pathogenesis of keratitis. Ocular Immunol Inflamm 2005;13(2-3):133-38.
21. Palomo J, Dietrich D, Martin P, Palmer G, Gabay C. The interleukin (IL)-1 cytokine family--Balance between agonists and antagonists in inflammatory diseases. Cytokine 2015;76(1):25-37.

22. Hazlett LD. Corneal response to Pseudomonas aeruginosa infection. Prog Retin Eye Res 2004;23(1):1-30.

23. Uchino E, Sonoda S, Kinukawa N, Sakamoto T. Alteration pattern of tear cytokines during the course of a day: diurnal rhythm analyzed by multicytokine assay. Cytokine 2006;33(1):36-40.

24. Thakur A, Willcox MD. Contact lens wear alters the production of certain inflammatory mediators in tears. Exper Eye Res 2000;70(3):255-59.

25. Torres PF, Kijlstra A. The role of cytokines in corneal immunopathology. Ocular Immunol Inflamm 2001;9(1):9-24.

26. Cavanagh HD, Ladage P, Yamamoto K, Li SL, Petroll WM, Jester JV. Effects of daily and overnight wear of hyper-oxygen transmissible rigid and silicone hydrogel lenses on bacterial binding to the corneal epithelium: 13-month clinical trials. Eye Contact Lens 2003;29 (1 Suppl):S14-16; discussion S26-19, S192-194.

27. Dogru M, Ward SK, Wakamatsu T, et al. The effects of 2 week senofilcon-A silicone hydrogel contact lens daily wear on tear functions and ocular surface health status. Contact Lens Anterior Eye 2011;34(2): 77-82.

28. Carnt NA, Willcox MD, Hau S, et al. Immune defense single nucleotide polymorphisms and recruitment strategies associated with contact lens keratitis. Ophthalmology 2012;119(10):1997-2002.

29. Carnt NA, Willcox MD, Hau S, et al. Association of single nucleotide polymorphisms of interleukins-1beta, -6 , and $-12 \mathrm{~B}$ with contact lens keratitis susceptibility and severity. Ophthalmology 2012;119(7):1320-27.

30. Keijser S, Kurreeman FA, de Keizer RJ, et al. IL-10 promotor haplotypes associated with susceptibility to and severity of bacterial corneal ulcers. Experiment Eye Research 2009;88(6):1124-28.

31. Xue ML, Thakur A, Lutze-Mann L, Willcox MD. Pro-inflammatory cytokine/chemokine gene expression in human corneal epithelial cells colonized by Pseudomonas aeruginosa. Clin Experiment Ophthalmol 2000;28(3):197-200.

J Cont Lens Res Sci Vol 1(1):3-21; September 29, 2017.

(c) 2017 Journal of Contact Lens Science and Research. All rights reserved. This article is distributed under the terms of the Creative Commons Attribution-Non Commercial 4.0 International License. 
32. Yu JJ, Ruddy MJ, Wong GC, et al. An essential role for IL-17 in preventing pathogen-initiated bone destruction: recruitment of neutrophils to inflamed bone requires IL-17 receptor-dependent signals. Blood 2007;109(9):3794-802.

33. Shahrara S, Pickens SR, Mandelin AM, 2nd, et al. IL17-mediated monocyte migration occurs partially through CC chemokine ligand 2/monocyte chemoattractant protein-1 induction. J Immunol 2010;184(8):4479-87.
34. Kalsow CM, Reindel WT, Merchea MM, Bateman KM, Barr JT. Tear cytokine response to multipurpose solutions for contact lenses. Clin Ophthalmol 2013;7:1291-302.

35. Markoulli M, Papas E, Cole N, Holden B. Effect of contact lens wear on the diurnal profile of matrix metalloproteinase 9 in tears. Opto Vision Science 2013;90(5):419-29.

36. Chao C, Golebiowski B, Stapleton F, Richdale K. Changes in tear cytokine concentrations following discontinuation of soft contact lenses-a pilot study. Eye Contact Lens 2016;42(4):237-43.

J Cont Lens Res Sci Vol 1(1):3-21; September 29, 2017. 